\title{
Correction to: A tailored approach in lymph node-positive perihilar cholangiocarcinoma
}

\author{
Christian Benzing ${ }^{1}$ (1) Felix Krenzien ${ }^{1} \cdot$ Alexa Mieg $^{1} \cdot$ Annika Wolfsberger $^{1} \cdot$ Andreas Andreou $^{1} \cdot$ Nora Nevermann $^{1}$. \\ Uwe Pelzer ${ }^{2}$. Uli Fehrenbach ${ }^{3} \cdot$ Lena Marie Haiden $^{1} \cdot$ Robert Öllinger $^{1} \cdot$ Wenzel Schöning $^{1} \cdot$ Moritz Schmelzle $^{1}$. \\ Johann Pratschke ${ }^{1}$
}

Published online: 31 October 2021

๑) Springer-Verlag GmbH Germany, part of Springer Nature 2021

\section{Correction to: Langenbeck's Archives of Surgery (2021) 406:1499-1509 https://doi.org/10.1007/s00423-021-02154-4}

Figures 3 and 4 did not precisely match the text and were incorrect. Figure 3 refers to the survival according to the side of hepatic resection and not R-status. In Figure 4, the reasons for left/right hepatectomy not being feasible were reversed.

Corrected figures and figure legends are shown below:
Figure 3: Overall and disease-free survival of patients with lymph node positive perihilar cholangiocarcinoma according to side of hepatic resection

Kaplan Meier curves of patients with lymph node metastases and resected perhilar cholangiocarcinoma according to side of hepatic resection. A. Overall survival. B.

The original article can be found online at https://doi.org/10.1007/ s00423-021-02154-4.

Moritz Schmelzle

moritz.schmelzle@charite.de

1 Department of Surgery, Campus Charité Mitte Campus Virchow-Klinikum, Experimental Surgery and Regenerative Medicine, Charité - Universitätsmedizin Berlin, Berlin, Germany

2 Department of Hematology, Oncology and Tumor Immunology, Charité - Universitätsmedizin Berlin, Berlin, Germany

3 Department of Radiology, Charité - Universitätsmedizin Berlin, Berlin, Germany 
Overall survival excluding 90-day mortality. C. Diseasefree survival. D. Disease-free survival excluding 90-day mortality. E. Overall survival after propensity score matching excluding 90-day mortality. F. Disease-free survival after propensity score matching excluding 90-day mortality.
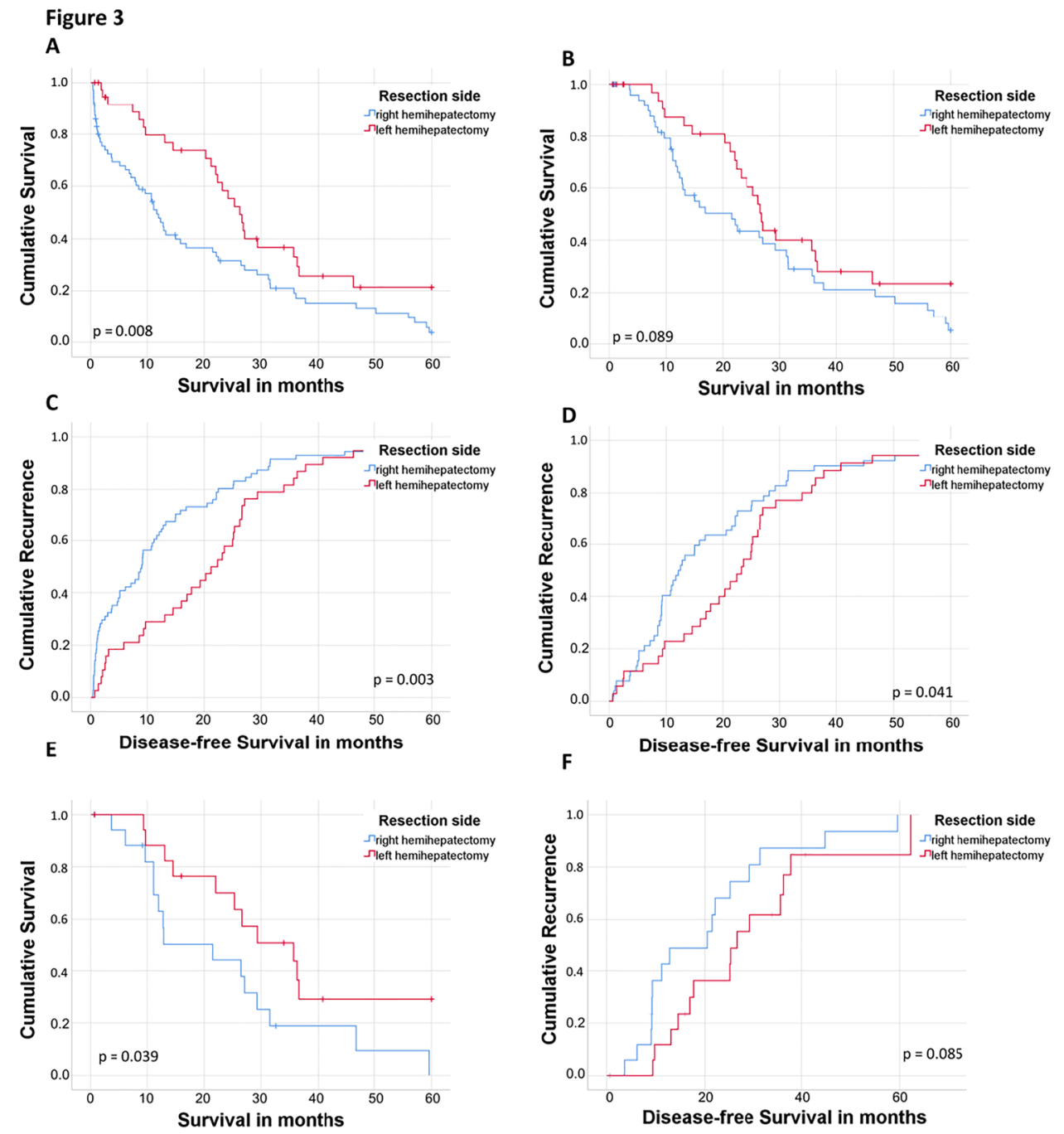
Figure 4: Suggested therapy algorithm in resectable perihilar cholangiocarcinoma
Suggested tailored approach in patients diagnosed with resectable perihilar cholangiocarcinoma without distant metastases (Figure created with Biorender.com)

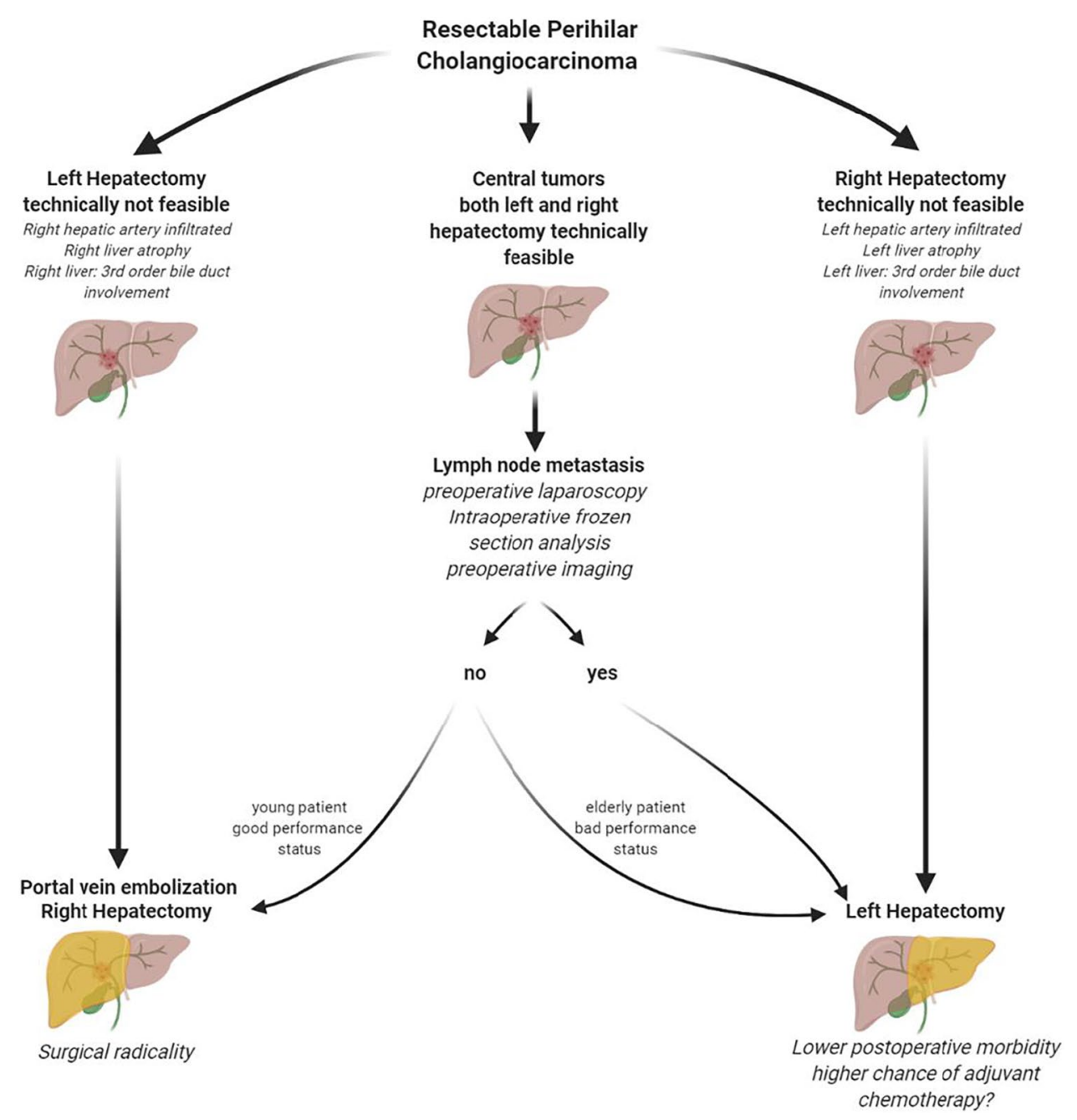

The original article has been corrected

Publisher's note Springer Nature remains neutral with regard to jurisdictional claims in published maps and institutional affiliations. 